\title{
Laser Induced Changes of the Raman Spectra of Pristine and Poly(tert-Butyl Acrylate) Functionalized Carbon Nanotubes
}

\author{
Deokjin Yu, Charles W. Blackledge, James P. Wicksted \\ Department of Physics, Oklahoma State University, Stillwater, Oklahoma, USA \\ Email: deokjin.yu@okstate.edu, cwblackledge@yahoo.com, james.wicksted@okstate.edu
}

Received 26 September 2013; revised 10 November 2013; accepted 10 December 2013

Copyright (C) 2014 by authors and Scientific Research Publishing Inc.

This work is licensed under the Creative Commons Attribution International License (CC BY). http://creativecommons.org/licenses/by/4.0/

(c) (i) Open Access

\begin{abstract}
Pristine and poly(tert-butyl acrylate) (PTBA) functionalized carbon nanotubes are continuously exposed to $2.41 \mathrm{eV}$ laser irradiation while collecting Raman spectra. The loss of the intensity of the radial breathing modes (RBMs) of small metallic PTBA functionalized nanotubes is less than that of pristine nanotubes. A reduction of the intensity of the $G^{-}$band of pristine SWNTs occurs such that the overall shape of the $G$ band evolves to resemble that of the PTBA functionalized sample. Complementing the measurement of the ratio of intensities of the $D$ and $G$ bands, the laser-induced spectral changes provide another way to determine the sidewall functionalization of carbon nanotubes. The laser-induced changes of the G and RBM bands are consistent with the greater sidewall reactivity of small metallic nanotubes toward functionalization with PTBA and reaction with photosensitized oxygen.
\end{abstract}

\section{Keywords}

Raman Spectroscopy, Functionalized Carbon Nanotubes

\section{Introduction}

Carbon nanotubes have received attention due to their interesting structure and electronic, mechanical, heat conductive, and other physical properties. The synthesis of carbon nanotubes often yields inhomogeneous mixtures with variable dimensions and symmetries that determine their electronic, vibronic, and other characteristics. Sample inhomogeneity is inconvenient for two reasons: scientifically it complicates the interpretation of experimental data such as Raman spectra; technologically it impedes progress in the applications of single walled carbon nanotubes (SWNTs) because many uses require scale-up and integration of ensembles. Solutions to the 
longstanding problem of sample inhomogeneity are sought by multiple methods as mentioned by Huang et al. [1] Sidewall functionalization has emerged as a possible route to select or separate carbon nanotubes from mixtures [2] [3] and the chemistry of carbon nanotube surface functionalization has been reviewed [4].

Raman spectroscopy is frequently employed in order to characterize both pristine and functionalized SWNTs. It obtains information about the fundamental electronic and vibrational structure, provides evidence of the selective oxidation of metallic carbon nanotubes [1] [5], and is useful as a convenient measure of sidewall functionalization [2]. In this work, the laser induced spectral changes of pristine and poly(tert-butyl acrylate) (PTBA) functionnalized SWNTs are compared under identical experimental conditions. Laser-induced losses of the higher frequency radial breathing modes (RBMs) of smaller SWNTs and the $\mathrm{G}^{-}$band of Raman spectra are compared and discussed for pristine and PTBA functionalized SWNTs. Complementing the measurement of the $D / G$ ratio that is used as evidence of sidewall functionalization [2], the analysis of the spectral changes of the RBMs and $\mathrm{G}$ band also provide a means to discriminate pristine and polymer functionalized carbon nanotubes.

\section{Experimental Section}

\subsection{Single-Walled Carbon Nanotubes and PTBA-SWNT}

SWNT were HiPco, batch number PO175, from Carbon Nanotechnologies Inc., Houston, TX. Grafting of poly(tert-butyl acrylate) was carried out by a procedure similar to that for grafting of poly(4-vinylpyridine) [6]. A $50 \mathrm{~mL}$ dried Schlenk flask was charged with a magnetic stirrer, $60 \mathrm{mg}$ of pristine SWNT, and $30 \mathrm{~mL}$ of $\mathrm{N}, \mathrm{N}$-dimethylformamide (DMF). After stirring for $12 \mathrm{~h}$ at room temperature, $6.0 \mathrm{~g}$ of tert-butyl acrylate and azobisisobutyronitrile ( $2 \mathrm{~mol} \%$ relative to tert-butyl acrylate) were added. The solution was stirred for 15 min at $0^{\circ} \mathrm{C}$. The mixture was degassed by three freeze-pump-thaw cycles. The flask was placed in an oil bath at $65^{\circ} \mathrm{C}$ for $48 \mathrm{~h}$ while stirring the mixture. The mixture was diluted to $200 \mathrm{~mL}$ with DMF and was centrifuged at $5000 \times$ $\mathrm{g}$ for $2 \mathrm{~h}$. The yellowish supernatant polymer solution was decanted. The black precipitate was redispersed in DMF by shaking, and centrifugation and decantation were repeated. The sediment was diluted to $200 \mathrm{~mL}$ with DMF, bathsonicated for $1 \mathrm{~h}$, and centrifuged at $5000 \times \mathrm{g}$ for $3 \mathrm{~h}$. After removal of the black supernatant solution, the sediment was redispersed in DMF by bath sonication for $10 \mathrm{~min}$, and the mixture was centrifuged at $5000 \times$ $\mathrm{g}$ for $3 \mathrm{~h}$. The supernatant was collected, and the procedure was repeated two more times. The dispersions still appeared black without visible precipitate after standing for 2 weeks. Removal of free polymer in the supernatant is necessary to speed the following ultrafiltration process. The combined black supernatant solutions were vacuum-filtered through a $0.2 \mu \mathrm{m}$ PTFE membrane. After washing with eight 40-mL portions of DMF, no cloudiness appeared when ten drops of filtrate was added to $15 \mathrm{~mL}$ of methanol, indicating that little or no soluble free polymer remained in the mixture. The black solid PTBA-SWNT collected on the membrane was dried in vacuum for $36 \mathrm{~h}$ at $50^{\circ} \mathrm{C}$. TGA under nitrogen up to $800^{\circ} \mathrm{C}$ gave a $30 \%$ weight loss due to the polymer. AFM showed small bundles of tubes with a range of lengths $0.3-3 \mu \mathrm{m}$, a range of heights of $3.0-10.3 \mathrm{~nm}$, and an average height of $6.5 \mathrm{~nm}$. FTIR showed a strong carbonyl band at $1723 \mathrm{~cm}^{-1}$. Samples were drop coated on silicon wafers.

\subsection{Raman Spectroscopy}

Raman was performed in backscattering geometry using an Argon ion laser, $514.5 \mathrm{~nm}$ (2.41 eV), at an intensity of approximately $20 \mathrm{~kW} / \mathrm{cm}^{2}$, focused through a 0.95 NA objective (Olympus). A Jobin-Yvon U1000 double grating spectrometer was used. Detection was with an electronically cooled photomultiplier tube (RCA 943-02). Data was taken with 1 second ( $G$ and D regions, $1650 \mathrm{~cm}^{-1}$ to $1450 \mathrm{~cm}^{-1}$ ) and 2 second (RBM region, $300 \mathrm{~cm}^{-1}$ to $150 \mathrm{~cm}^{-1}$ ) integration times per data point.

The peak areas in Raman spectra were calculated by numerical integration using baseline subtractions common to each spectral region (RBM or G) of each sample set (pristine or PTBA functionalized) with one exception. The baseline for the spectrum of the RBM region of the pristine sample taken at 40 min was found independently due to the presence of significantly more background light. The baseline was calculated from the average of the first and last eight data points in each spectral range. The area of the metallic RBMs, $R B M_{m}$, was calculated by numerical integration of the range $240-300 \mathrm{~cm}^{-1}$, and this is normalized to the area from 150 $\mathrm{cm}^{-1}$ to $300 \mathrm{~cm}^{-1}, R B M_{t}$. The area of the $\mathrm{G}^{-}$band, $\mathrm{G}^{-}$, was calculated by numerical integration of the range 1450 $-1576 \mathrm{~cm}^{-1}$, and was normalized to the area from $1450 \mathrm{~cm}^{-1}$ to $1650 \mathrm{~cm}^{-1}, \mathrm{G}_{\mathrm{t}}$. 


\section{Results}

Figure 1 shows the Raman spectra of the $D\left(1330 \mathrm{~cm}^{-1}\right)$ and $\mathrm{G}$ regions $\left(1450-1630 \mathrm{~cm}^{-1}\right)$ of pristine and PTBA functionalized SWNTs after background subtraction and normalization to the maximum intensity of the $\mathrm{G}$ mode. The ratio of the maximum intensity of the $\mathrm{D}$ and $\mathrm{G}$ bands $(D / G)$ is 0.24 for the pristine sample, and 0.36 for the PTBA functionalized one. Acquiring spectra from multiple positions on the same sample, $D / G$ can be calculated to within an error of less than 5\% conservatively, and usually less than $1.5 \%$.

In Figure 2A, the initial and final Raman spectra of the RBM and G regions of a pristine sample that is subjected to constant irradiation at approximately $20 \mathrm{~kW} / \mathrm{cm}^{2}$ for $40 \mathrm{~min}$ are shown. The spectra of a PTBA functionalized sample that is subjected to identical conditions appear in Figure 2B. The spectra are not normalized. For both samples, a significant loss in the intensity of the radial breathing modes that lie in the range 240 - 290 $\mathrm{cm}^{-1}$ is discerned when compared with the nearly constant intensity of the modes at $209 \mathrm{~cm}^{-1}$ and $188 \mathrm{~cm}^{-1}$. In addition to the loss of intensity of the metallic RBMs, a loss of intensity of the $\mathrm{G}^{-}$band is observed in the pris-

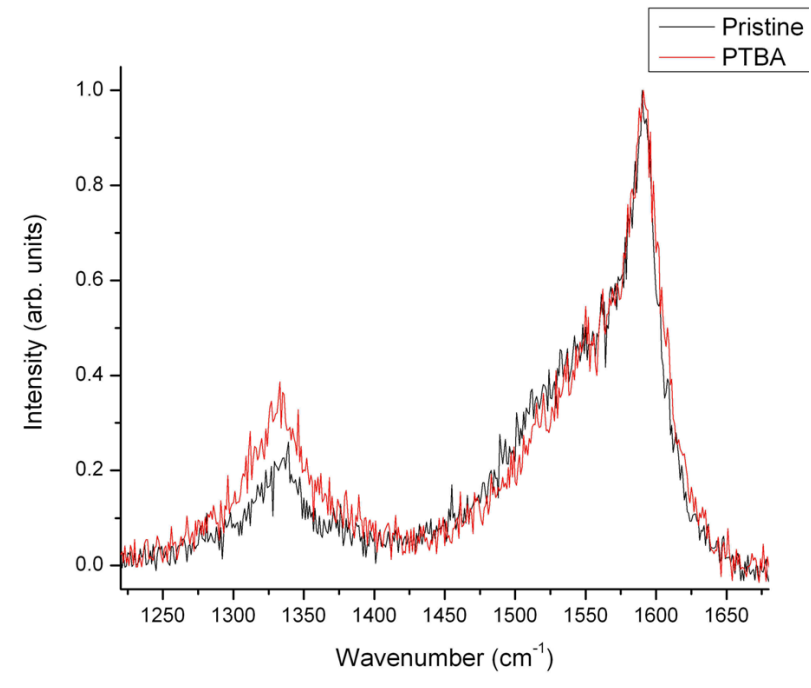

Figure 1. Raman spectra of pristine and PTBA functionalized SWNTs. The $D / G$ ratios are 0.24 and 0.36 , respectively. The greater intensity of the $\mathrm{G}^{-}$shoulder $\left(1450-1576 \mathrm{~cm}^{-1}\right)$ of the pristine sample compared to the PTBA sample can also be faintly discerned. Spectra are normalized to the peak intensity of the $\mathrm{G}^{+}$mode at $1590 \mathrm{~cm}^{-1}$.
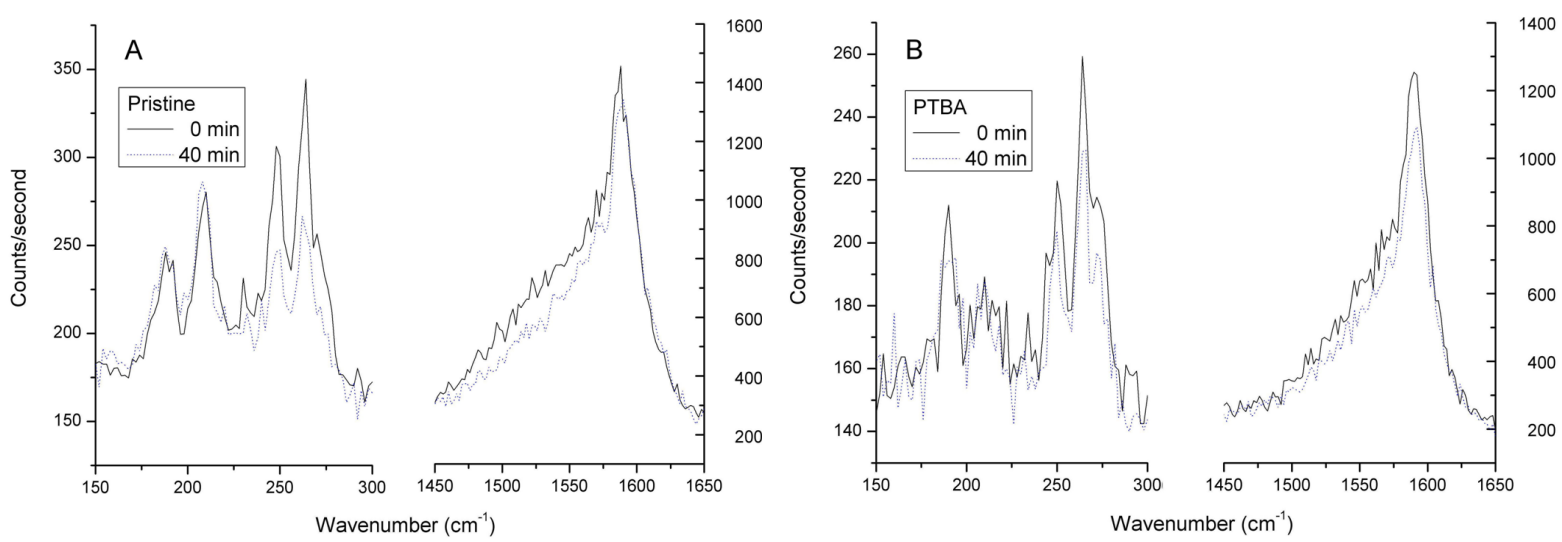

Figure 2. Raman spectra of the RBM and G regions of a pristine (A) and PTBA functionalized (B) sample before and after $40 \mathrm{~min}$ of laser irradiation. The intensity of the RBMs of metallic SWNTs in the range $240-290 \mathrm{~cm}^{-1}$ is reduced over time. The reduction of the intensity of the $\mathrm{G}^{-}$band that lies in the range $1450-1578 \mathrm{~cm}^{-1}$ is also clearly discerned in the pristine sample, but this effect is much smaller in the PTBA sample. Spectra are not normalized. 
tine sample (Figure 2A).

The ratio of the spectral area of the metallic RBM to the total RBM, $R B M_{m} / R B M_{t}$, is plotted versus time (Figure 3). Plots of the ratio $R B M_{m}(t) / R B M_{t}(t)$ rather than $R B M_{m}(t) / R B M_{m}(t=0)$ are shown because they yielded better fits to single exponential functions $\left(I=A e^{-t /}\right)$. A normalization procedure such as the one used here is necessary to correct for fluctuations and drift of laser power that occur over the time scale of the experiment.

The integrated spectral area of the $\mathrm{G}^{-}$band is normalized $\left(\mathrm{G}^{-} / \mathrm{G}_{\mathrm{t}}\right)$ and plotted in Figure 4. Over 40 minutes, the reduction of the normalized area of the $\mathrm{G}^{-}$band of the PTBA functionalized sample is only $2.9 \%$ compared to the $12 \%$ of the pristine sample. The functionalization of the sidewalls with PTBA decreases the extent of subsequent laser-induced change of the Raman intensities of the RBM and G regions. Laser assisted spectral changes of the metallic RBMs follow a similar trend, yielding a normalized intensity of $R B M_{m}$ of PTBA functionalized samples of $8.7 \%$, and for pristine samples, $23 \%$. The key spectrally based measurements are shown in Table 1. Estimates of the diameters of nanotubes, from the equation $d_{t}=248 / \omega\left(d_{t}\right.$, diameter in nm; $\omega$, RBM frequency in $\mathrm{cm}^{-1}$ ) [7], and tentative assignments of chiral indices $(n, m)$ are given in Table 2 [1].

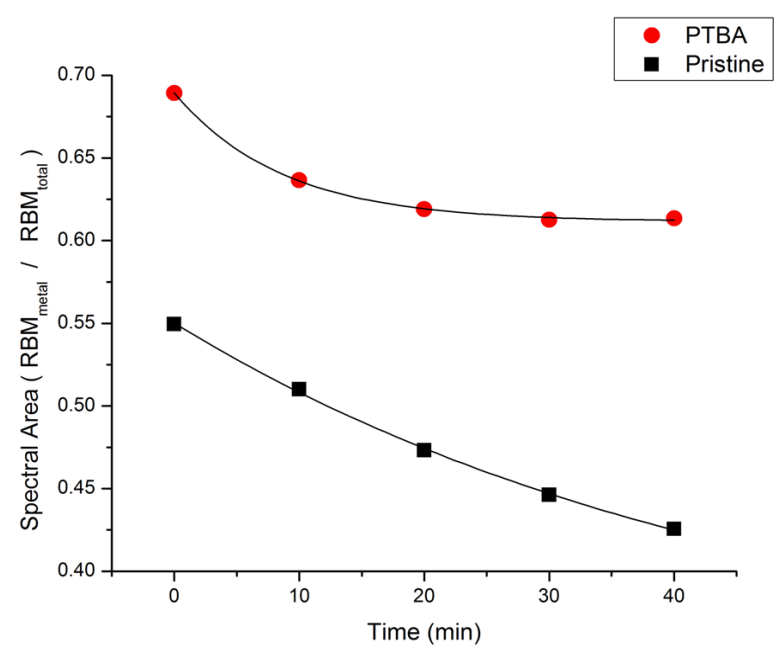

Figure 3. Plots of the normalized areas of RBMs of metallic SWNTs of PTBA functionalized (circles) and pristine (squares) samples versus time of continuous laser irradiation ( $\left.20 \mathrm{~kW} / \mathrm{cm}^{2}\right)$.

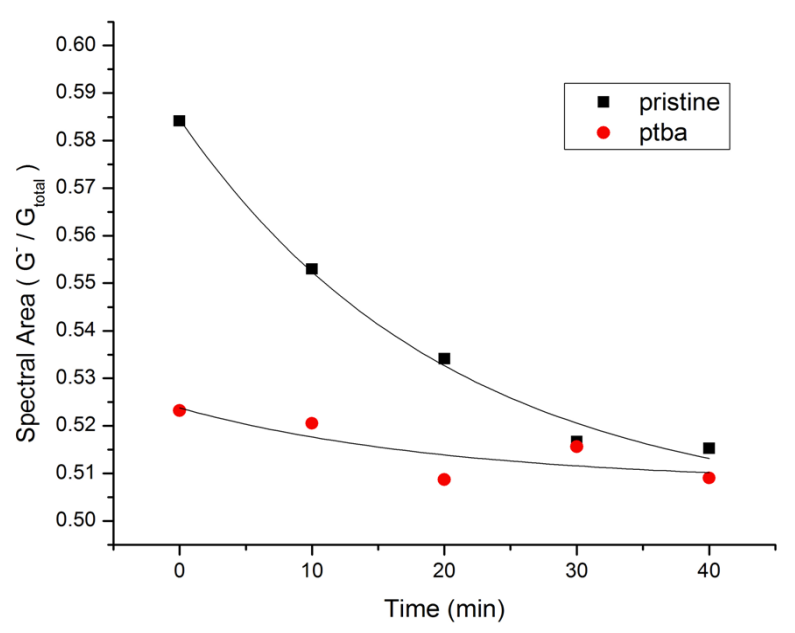

Figure 4. Plots of the normalized areas of the $\mathrm{G}^{-}$bands of PTBA functionalized (circles) and pristine (squares) carbon nanotube samples versus time of continuous laser irradiation $\left(\sim 20 \mathrm{~kW} / \mathrm{cm}^{2}\right)$. 
Table 1. The easily measurable empirical differences between the Raman spectra of pristine and PTBA functionalized SWNTs.

\begin{tabular}{cccc}
\hline Sample & $D / G$ & $\Delta\left(\mathrm{G}^{-} / \mathrm{G}_{\mathrm{t}}\right)$ & $\Delta\left(R B M_{m} / R B M_{t}\right)$ \\
\hline Pristine & $24 \%$ & $23 \%$ & $12 \%$ \\
PTBA & $36 \%$ & $8.7 \%$ & $2.9 \%$ \\
\hline
\end{tabular}

\begin{tabular}{ccc}
\multicolumn{3}{c}{ Table 2. Tentative assignments of chiral indices. } \\
\hline RBM frequency $\left(\mathrm{cm}^{-1}\right)$ & Diameter $(\mathrm{nm})$ & Assignment $(n, m)$ \\
271 & 0.92 & metal $(9,3)$ \\
263 & 0.94 & metal $(8,5)$ \\
248 & 1.0 & metal $(12,0)$ \\
209 & 1.2 & semiconductor $(12,4)$ \\
188 & 1.3 & semiconductor $(16,0)$ \\
\hline
\end{tabular}

\section{Discussion}

\subsection{Discrimination of Pristine and Sidewall Functionalized Nanotubes}

The increase in $D / G$ after the PTBA treatment shown in Figure 1 is an indicator of the chemical attachment of the PTBA group to the nanotube sidewall [2]. Sidewall functionalization is expected to increase the density of sidewall defect-sites that break the nanotube symmetry [2], and increase the intensity of the $\mathrm{D}$ band. The reaction of $\mathrm{sp}^{2}$ carbons to form $\mathrm{sp}^{3}$ carbons that occurs upon sidewall functionalization should not be interpreted as a necessary condition for an increase in the intensity of the $\mathrm{D}$ band, although the double resonance mechanism that accounts for the $\mathrm{D}$ band requires a defect site to satisfy the conservation of momentum during the Raman scattering process [8]. The spectral changes that accompany sidewall functionalization are sometimes assumed to be entirely due to an increase of the intensity of the $\mathrm{D}$ band that is directly caused by increase of the density of $\mathrm{sp}^{3}$ defects in the sidewalls.

Spectra are commonly normalized to the maximum intensity of the $\mathrm{G}$ band in order to ease the comparison of different spectra. The changes to $D / G$ after sidewall functionalization can thus be possibly due to changes occurring to both the $D$ and $G$ regions. Notably, sidewall functionalization with PTBA changes the shape of the $G$ band, reducing the relative intensity of the $\mathrm{G}^{-}$band which is the low frequency shoulder of the $\mathrm{G}$ band from $1450-1576 \mathrm{~cm}^{-1}$.

During continuous laser irradiation of pristine carbon nanotubes, spectral changes occur that affect the $D / G$ ratio [9] as well as the intensities of the radial breathing modes [10] [11], although no significant changes to the intensity of the $\mathrm{D}$ band are observed. The $\mathrm{G}^{-}$region of pristine carbon nanotubes undergoes a significant reduc tion of intensity that is greater than that of the PTBA functionalized samples. The shape of the entire $G$ band of pristine nanotubes evolves under constant irradiation to resemble that of the PTBA functionalized nanotubes. The RBMs that arise from small metallic nanotubes of both pristine and PTBA functionalized samples also undergo a noticeable reduction of intensity. The laser-induced spectral changes that we observed are not reversed after samples are left overnight in the dark. Table 1 summarizes the easily measurable empirical differences between the Raman spectra of pristine and PTBA functionalized SWNTs.

\subsection{Radial Breathing Modes}

The higher frequency RBMs at $271 \mathrm{~cm}^{-1}, 263 \mathrm{~cm}^{-1}$, and $248 \mathrm{~cm}^{-1}$ are assigned to metallic SWNTs, and the lower frequency modes at $209 \mathrm{~cm}^{-1}$ and $188 \mathrm{~cm}^{-1}$ are assigned to semiconductor SWNTs that are larger in diameter than the metallic SWNTs [1]. The chiral index $(n, m)$ determines its electronic structure and only the nanotubes that have a difference in electronic band energies near the laser's energy of $2.41 \mathrm{eV}$ provide a significant Raman scattering signal. 
The two prominent semiconductor bands at $188 \mathrm{~cm}^{-1}$ and $209 \mathrm{~cm}^{-1}$ swap dominance after sidewall functionalization as seen by comparing the low frequency RBM regions of Figure 2A and Figure 2B. This may be ascribed to a greater susceptibility of the $209 \mathrm{~cm}^{-1}$ nanotubes to sidewall functionalization that is determined by the chiral index $(n, m)$, noting that the bond strain, diameter, and density of states near the Fermi level of pristine SWNTs can all be theoretically calculated from the chiral index.

The effect of sidewall functionalization with PTBA on the peaks at $188 \mathrm{~cm}^{-1}$ and $209 \mathrm{~cm}^{-1}$ may also be ascribed to two other contributing factors even if every nanotube type $(n, m)$ exhibits the same reactivity. 1$)$ Upon sidewall functionalization with PTBA, the RBM of the $209 \mathrm{~cm}^{-1}$ nanotube undergoes a greater loss in polarizability, $\alpha$, thereby reducing its Raman cross-section, compared to the $188 \mathrm{~cm}^{-1}$ nanotube. In other words, $\mathrm{d} \alpha / \mathrm{dN}$, with $\mathrm{dN}=$ the differential change in the surface density of functionalization attachment points, is more negative for the $209 \mathrm{~cm}^{-1}$ nanotube than the $188 \mathrm{~cm}^{-1}$ nanotube. 2) Upon sidewall functionalization, the electronic states of the $209 \mathrm{~cm}^{-1}$ nanotube undergo a shift in energy that brings the nanotube farther out of resonance with the excitation laser in comparison to the $188 \mathrm{~cm}^{-1}$ nanotube. Sidewall functionalization alters the electronic band structure of SWNTs [12]. This may increase or decrease the electronic resonance of the SWNTs with the 2.41 $\mathrm{eV}$ laser, depending on the nanotube type $(n, m)$. In other words, the nanotube symmetry $(n, m)$ determines how great of a shift of each electronic band occurs after the nanotube sidewall is derivatized. The sensitivity of the energy of an electronic band to surface functionalization may be expressed as $\mathrm{dE} / \mathrm{dN}$, with $\mathrm{dE}=$ the differential change in the energy of an electronic band. Each nanotube type $(n, m)$ may have a unique value of $\mathrm{dE} / \mathrm{dN}$ for each of its electronic bands. The relative increase of the intensity of the $188 \mathrm{~cm}^{-1} \mathrm{RBM}$ compared to the 209 $\mathrm{cm}^{-1} \mathrm{RBM}$ that occurs upon functionalization (left side of Figure 1A and Figure 1B) may be attributed partly to such a mechanism.

The initial relative spectral area of the metallic RBMs (at $t=0$ in Figure 3 ) is significantly greater for the PTBA functionalized nanotubes (0.69) than the pristine nanotubes $(0.55)$. This is due to the relative reduction, after functionalization, of the intensity of the semiconductor mode at $209 \mathrm{~cm}^{-1}$ (Figure 2A and Figure 2B).

Over time, the spectral areas of the semiconductor RBMs of the pristine and PTBA functionalized samples do not change significantly as they are subjected to constant irradiation. In contrast, the loss of the intensity of the higher frequency metallic RBMs has been attributed to sample oxidation by reaction with singlet oxygen that is generated from the atmosphere [10]. Singlet oxygen can be generated by photoexcited impurities such as buckeyballs or left-over metal from the catalyst particles that are used in synthesis. Singlet oxygen binds to structurally perfect SWNTs [13], and forms endoperoxides that change the SWNT band structure [14]. Further irreversible oxidative steps are also likely.

Generally, small diameter SWNTs are more reactive than large diameter ones due to the greater bond strain of small diameter SWNTs. This alone may account for the greater loss of the intensity of the higher frequency (metallic) RBMs compared to the lower frequency (semiconductor) ones [15], although nanotube symmetries $(n, m)$ also contribute to the sidewall reactivity [1] [16]. The high density of states near the Fermi level of metallic nanotubes which are identified with chiral indices $(n, m)$ such that $2 n+m$ is divisible by 3 contributes to their enhanced reactivity in comparison to semiconductor nanotubes.

The decay of the Raman intensity of the metallic RBM region of the PTBA functionalized sample is significantly faster compared to the pristine one (Figure 3). The decay times obtained are: $8.7 \pm 0.5 \mathrm{~min}$ for the PTBA sample, and $47 \pm 7 \mathrm{~min}$ for the pristine sample. The difference in rates is attributed to a reduction of the thermal conductivity of PTBA functionalized samples compared to pristine ones.

The thermal conductivity of carbon nanotubes is unusually high [12] [17], and is reduced after sidewall functionalization for two reasons: functionalization increases the scattering of phonons along the nanotubes which decreases the thermal conductivity, and the polymer moiety has a lower thermal conductivity than the carbon nanotubes by a factor of approximately 1000. Compared to the pristine SWNTs, the PTBA functionalized nanotubes are separated, with a polymer that has a much lower thermal conductivity that fills the space between them. The pristine SWNTs conduct heat away from the irradiated spot more quickly, thus they oxidize more slowly.

\subsection{G Band}

The G band arises from vibrational modes that are oriented tangentially to the surface of the carbon nanotube. In each carbon nanotube type $(n, m)$, six vibrational modes are present across the entire range of the $G$ band, and at least half are Raman active in each type of nanotube [7] [12]. The G band is separated into a narrower band at 
$1590 \mathrm{~cm}^{-1}\left(\mathrm{G}^{+}\right)$that is not as sensitive to the symmetry and diameter of the nanotube as is the broader component, the $\mathrm{G}^{-}$band. The many types of electronically resonant SWNTs present in samples have $\mathrm{G}^{-}$frequencies and intensities that are dependent on their symmetry. The observed intensity ratio, $G^{-} / G_{t}$, of the Raman peaks of isolated nanotubes is variable [7], and depends on the nanotube's electronic band structure which determines its resonance Raman enhancement.

Under constant irradiation, the loss of intensity of the $\mathrm{G}^{-}$band of the pristine sample occurs such that the shape of its entire $G$ band approaches that of the functionalized sample (Figure 4). The laser induced effects on the $\mathrm{G}$ band of pristine nanotubes is apparently the same as the attachment of PTBA, that is, to reduce the $\mathrm{G}^{-}$ component. Both sidewall functionalization with PTBA and reaction with ${ }^{1} \mathrm{O}_{2}$ occur more readily to the same types of nanotubes that provide the $\mathrm{G}^{-}$Raman signal of the overall resonant set that provide the entire resonant Raman signals.

Among the nanotubes that provide a Raman signal, the set of pristine nanotubes that have a relatively high $\mathrm{G}^{-} / \mathrm{G}_{\mathrm{t}}$ ratio are, observationally, the most susceptible to oxidation and sidewall functionalization with PTBA. These may be regarded as metallic nanotubes because of the asymmetric Fano shaped contribution of metallic SWNTs to the low frequency $\mathrm{G}^{-}$shoulder of the $\mathrm{G}$ band. In addition to the direct reaction of the sidewalls of the SWNTs, the laser-induced loss of the intensity of the $\mathrm{G}^{-}$band of pristine SWNTs may also be attributed to heat-induced separation, and an accompanying reduction of plasmon coupling of separated SWNTs that reduces the $\mathrm{G}^{-}$signal. Debundling causes a loss of electronic coupling between neighbors [18], and blue-shifts the electronic absorption [19]. Such effects are not observed in PTBA functionalized SWNTs because by attaching polymers to the sidewalls, the nanotube bundles have become separated from each other. Even after drying the sample on a substrate, the presence of PTBA on the sidewalls increases the center-to-center distance between neighboring nanotbues, and reduces the electronic coupling between neigh-boring nanotubes. Bundling is reduced in the PTBA functionalized sample, and the extent of laser-assisted change of its $\mathrm{G}^{-}$band is less than that of the pristine sample.

\subsection{Comparison of Laser-Induced Changes of the G and RBM Bands}

Evidence for the selective laser-induced oxidation of small carbon nanotubes based on resonance Raman spectroscopy of the RBM region is substantial [9] [10] [15]. Unfortunately, the resonance conditions for the RBMs and $\mathrm{G}$ band differ by $\sim 0.2 \mathrm{eV}$, so a different set of carbon nanotubes provides the signal in these two spectral regions [20]. There is no correlation between the rates of the changes in the RBM and G regions. There is correlation between the sample type (pristine versus PTBA functionalized) and the extent of laser-assisted change of $\mathrm{G}^{-} / \mathrm{G}_{\mathrm{t}}$ and $R B M_{m} / R B M_{t}$. In both spectral regions, greater changes are observed in the pristine sample compared to the PTBA functionalized sample as is shown in Table 1. Although the laser-induced changes of the $\mathrm{G}$ band may be ascribed to the preferential oxidation of small metallic SWNTs, the tangential modes of many types of SWNTs overlap to form the $\mathrm{G}^{-}$band. Consequently, the oxidation of other types of nanotubes is difficult to entirely discount.

\subsection{Effect of Sidewall Reactions on Raman Spectra}

Pristine nanotubes have a $\pi$-conjugated bond structure that is highly polarizable. Both sidewall addition and oxidation result in $\mathrm{sp}^{3}$ hybridized defect sites in the otherwise $\mathrm{sp}^{2}$ hybridized $\pi$-conjugated network, resulting in a decrease of the polarizability. Structural changes to the sidewalls are also expected to shift the electronic states, thereby altering the resonance enhancement of the Raman scattering. However, if a shift of the electronic bands were the dominant effect, then one would expect some nanotube types $(n, m)$ to be brought closer to resonance with the excitation laser and the resonance Raman cross-section of some vibrational modes to increase. For the laser-induced changes, this is not observed. The spectra of Figure 2 and the kinetic plots of Figure 3 and Figure 4 show the intensities of Raman modes either remaining constant or decreasing over time.

The laser-induced spectral changes of the RBMs of pristine samples signify preferential oxidation of small metallic SWNTs [1], and the data is also consistent with the greater susceptibility of small metallic SWNTs to sidewall functionalization with PTBA [2]. Reaction of tert-butyl acrylate or ${ }^{1} \mathrm{O}_{2}$ with the SWNT sidewalls causes the density of $\mathrm{sp}^{3}$ hybridized carbons to increase, the bond polarizability to decrease, and the (non-resonantly enhanced) Raman cross-section to decrease. The laser-induced spectral changes of the PTBA functionalized nanotubes are less than those of the pristine SWNTs because of two possible reasons. First, the Raman cross- 
section of functionalized SWNTs is already reduced. A further substantial increase in the density of $\mathrm{sp}^{3}$ sites by laser-assisted oxidation of the PTBA functionalized SWNTs that already have more $\mathrm{sp}^{3}$ defect sites than pristine SWNTs is not observed because of an already low Raman cross-section. The first possible reason does not invoke the second reason, which is that the functionalization of SWNTs reduces their reactivity to oxygen. The second reason is supported by the notion that oxygen binds more favorably to structurally perfect SWNTs [13], and less favorably to those with a greater defect density. Both reasons are possible.

\section{Conclusion}

Differences in the Raman spectra of pristine and PTBA functionalized SWNTs are reported. The reaction of SWNT sidewalls introduces $\mathrm{sp}^{3}$ hybridized defects into the largely $\mathrm{sp}^{2}$ hybridized bond structure. This decreases the polarizability of vibrational modes to reduce the Raman cross-section, and causes changes to the electronic band structure that may increase or decrease the Raman cross-section by altering the resonance enhancement. The functionalization of SWNTs with PTBA causes an increase of $D / G$ [2], and a decrease of subsequent laserinduced loss of intensity of RBMs of small metallic SWNTs and the $\mathrm{G}^{-}$band. The RBMs of small metallic pristine SWNTs undergo a loss of intensity that has been previously attributed to laser-assisted oxidation [1]. The loss of $R B M_{m} / R B M_{t}$ is greater in pristine SWNTs than PTBA functionalized SWNTs. The $\mathrm{G}^{-}$band of pristine samples is reduced by laser irradiation such that the shape of the entire $G$ band approaches that of the PTBA functionalized sample, and this is also attributable to the greater reactivity of smaller diameter metallic SWNTs which have greater bond strain and greater density of states near the Fermi level. Lastly, we note that laser induced oxidation occurs more slowly in pristine samples compared to PTBA functionalized samples due to the greater thermal conductivity of pristine SWNTs, which is responsible for removing heat from the focused spot.

\section{References}

[1] Huang, H., Maruyama, R., Noda, K., Kajiura, H. and Kadono, K. (2006) Preferential Destruction of Metallic SingleWalled Carbon Nanotubes by Laser Irradiation. The Journal of Physical Chemistry B, 110, 7316-7320. http://dx.doi.org/10.1021/jp056684k

[2] Strano, M.S., Dyke, C.A., Usrey, M.L., Barone, P.W., Allen, M.J., Shan, H.W., Kittrell, C., Hauge, R.H., Tour, J.M. and Smalley, R.E. (2003) Electronic Structure Control of Single-Walled Carbon Nanotube Functionalization. Science, 301, 1519-1522. http://dx.doi.org/10.1126/science.1087691

[3] Banerjee, S., Hemraj-Benny, T. and Wong, S.S. (2005) Routes Towards Separating Metallic and Semiconducting Nanotubes. Journal of Nanoscience and Nanotechnology, 5, 841-855. http://dx.doi.org/10.1166/jnn.2005.173

[4] Tasis, D., Tagmatarchis, N., Bianco, A. and Prato, M. (2006) Chemistry of Carbon Nanotubes. Chemical Reviews, 106, 1105-1136. http://dx.doi.org/10.1021/cr050569o

[5] Miyata, Y., Maniwa, Y. and Kataura, H. (2006) Selective Oxidation of Semiconducting Single-Wall Carbon Nanotubes by Hydrogen Peroxide. The Journal of Physical Chemistry B, 110, 25-29. http://dx.doi.org/10.1021/jp055692y

[6] Qin, S., Qin, D., Ford, W.T., Herrera, J.E. and Resasco Daniel, E. (2004) Grafting of Poly(4-vinylpyridine) to SingleWalled Carbon Nanotubes and Assembly of Multilayer Films. Macromolecules, 37, 9963-9967. http://dx.doi.org/10.1021/ma048692p

[7] Jorio, A., Souza Filho, A.G., Dresselhaus, G., Dresselhaus, M.S., Swan, A.K., Ünlü, M.S., Goldberg, B.B., Pimenta, M.A., Hafner, J.H., Lieber, C.M. and Saito, R. (2002) G-Band Resonant Raman Study of 62 Isolated Single-Wall Carbon. Physical Review B, 65, 155412.

[8] Thomsen, C. and Reich, S. (2000) Double Resonant Raman Scattering in Graphite. Physical Review Letters, 85, 5214-5217. http://dx.doi.org/10.1103/PhysRevLett.85.5214

[9] Osswald, S., Flahaut, E. and Gogotsi, Y. (2006) In Situ Raman Spectroscopy Study of Oxidation of Double- and Single-Wall Carbon Nanotubes. Chemistry of Materials, 18, 1525-1533. http://dx.doi.org/10.1021/cm052755g

[10] Bokova, S.N., Konov, V.I., Obraztsova, E.D., Osadchii, A.V., Pozharov, A.S. and Terekhov, S.V. (2003) Laser-Induced Effects in Raman Spectra of Single-Wall Carbon Nanotubes. Quantum Electronics, 33, 645. http://dx.doi.org/10.1070/QE2003v033n07ABEH002472

[11] Corio, P., Santos, P.S., Pimenta, M.A. and Dresselhaus, M.S. (2002) Evolution of the Molecular Structure of Metallic and Semiconducting Carbon Nanotubes under Laser Irradiation. Chemical Physics Letters, 360, 557-564. http://dx.doi.org/10.1016/S0009-2614(02)00866-7

[12] Burghard, M. (2005) Electronic and Vibrational Properties of Chemically Modified Single-Wall Carbon Nanotubes. 
Surface Science Reports, 58, 1-109.

[13] Savage, T., Bhattacharya, S., Sadanadan, B., Gaillard, J., Tritt, T.M., Sun, Y.P., Wu, Y., Nayak, S., Car, R., Marzari, N., Ajayan, P.M. and Rao, A.M. (2003) Photoinduced Oxidation of Carbon Nanotubes. Journal of Physics: Condensed Matter, 15, 5915-5921. http://dx.doi.org/10.1088/0953-8984/15/35/301

[14] Dukovic, G., White, B.E., Zhou, Z., Wang, F., Jockusch, S., Steigerwald, M.L., Heinz, T.F., Friesner, R.A., Turro, N.J. and Brus, L.E. (2004) Reversible Surface Oxidation and Efficient Luminescence Quenching in Semiconductor SingleWall Carbon Nanotubes. Journal of the American Chemical Society, 126, 15269-15276. http://dx.doi.org/10.1021/ja046526r

[15] Menna, E., Negra, F.D. and Fontana, M.D. (2003) Selectivity of Chemical Oxidation Attack of Single-Wall Carbon Nanotubes in Solution. Physical Review B, 68, 193412. http://dx.doi.org/10.1103/PhysRevB.68.193412

[16] Yang, C.-M., Park, J.S., An, K.H., Lim, S.C., Seo, K., Kim, B., Park, K.A., Han, S., Park, C.Y. and Lee, Y.H. (2005) Selective Removal of Metallic Single-Walled Carbon Nanotubes with Small Diameters by Using Nitric and Sulfuric Acids. Journal of Physical Chemistry B, 109, 19242-19248. http://dx.doi.org/10.1021/jp053245c

[17] Berber, S., Kwon, Y.-K. and Tom $\tilde{A}_{j}$ nek, D. (2000) Unusually High Thermal Conductivity of Carbon Nanotubes. Physical Review Letters, 84, 4613-4616. http://dx.doi.org/10.1103/PhysRevLett.84.4613

[18] Jiang, C.Y., Kempa, K., Zhao, J.L., Schlecht, U., Kolb, U., Basche, T., Burghard, M. and Mews, A. (2002) Strong Enhancement of the Breit-Wigner-Fano Raman Line in Carbon Nanotube Bundles Caused by Plasmon Band Formation. Physical Review B, 66, 161404. http://dx.doi.org/10.1103/PhysRevB.66.161404

[19] O’Connell, M.J., Sivaram, S. and Doorn, S.K. (2004) Near-Infrared Resonance Raman Excitation Profile Studies of Single-Walled Carbon Nanotube Intertube Interactions: A Direct Comparison of Bundled and Individually Dispersed HiPco Nanotubes. Physical Review B, 69, 235415.

[20] Saito, R., Jorio, A., Hafner, J.H., Lieber, C.M., Hunter, M., McClure, T., Dresselhaus, G. and Dresselhaus, M.S. (2001) Chirality-Dependent G-Band Raman Intensity of Carbon Nanotubes. Physical Review B, 6408, 085312. 